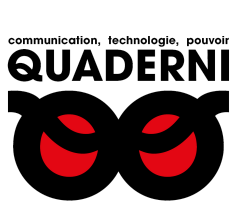

\title{
Quaderni
}

Communication, technologies, pouvoir

98 | Hiver 2018-2019

Humanités numériques : vers l'institutionnalisation

\section{Créativité, éducation aux médias et à l'information, translittératie : vers des humanités numériques}

Formerly, creativity was not a pedagogical priority

Divina Frau-Meigs

\section{OpenEdition}

1 Journals

Édition électronique

URL : https://journals.openedition.org/quaderni/1482

DOI : 10.4000/quaderni. 1482

ISSN : 2105-2956

Éditeur

Les éditions de la Maison des sciences de l'Homme

Édition imprimée

Date de publication : 5 février 2019

Pagination : 87-105

Référence électronique

Divina Frau-Meigs, « Créativité, éducation aux médias et à l'information, translittératie : vers des humanités numériques », Quaderni [En ligne], 98 | Hiver 2018-2019, mis en ligne le 05 février 2021, consulté le 07 janvier 2022. URL : http://journals.openedition.org/quaderni/1482 ; DOI : https:// doi.org/10.4000/quaderni. 7482 


\section{$D$ ossier}

\section{Créativité,}

éducation aux

médias et à

l'information,

translittératie :

vers des

humanités

numériques

\author{
Divina \\ Frau-Meigs
}

Professeur

Université Sorbonne Nouvelle
Dans les formes d'éducation pré-numériques, issues du XIX ${ }^{\mathrm{e}}$ siècle, la créativité n'était pas la priorité. Elles étaient focalisées sur des besoins liés à la deuxième révolution industrielle, nécessitant des ouvriers capables de faire preuve d'imitation, de production à l'identique à l'ère de la reproduction mécanique ${ }^{1}$. Les alphabétisations attenantes ont privilégié la lecture, l'écriture et le calcul comme compétences de base. La créativité s'est réfugiée dans une vision idéalisée de l'artiste non conformiste, échappé du système, et dans des approches non-disciplinaires, hors programmes scolaires. Souvent qualifiées d' " éducation à », fondées sur des pédagogies de l'action ou du projet, elles sont minoritaires ou marginalisées. Elles sont souvent associées à la prise en main d'outils difficiles à intégrer dans la salle de classe, comme dans le cas de la presse à imprimer pour l'éducation aux médias chère à Célestin Freinet.

$\mathrm{Au} \mathrm{XXI}^{\mathrm{e}}$ siècle, la troisième révolution industrielle a besoin d'ouvriers dans ses « forges » et ses « fabeliers » capables de faire preuve d'innovation, de création et non de copie, d'où la grande effervescence autour de la créativité, comme un état d'esprit associé à la production différenciée, non à l'identique, de l'ère numérique. L'artiste se réincarne sous la figure du « geek » ou de l'inventeur de génie capable de résoudre des problèmes inédits. Pour favoriser ce phénomène de créativité, de nouvelles littératies sont nécessaires qui supplantent la notion d'alphabétisation car il s'agit désormais de nouvelles pratiques de l'écrit, du lu et du calculé, qui articulent une technologie, la connaissance de celle-ci et des savoir-faire particuliers. Les « éducations à » trouvent tout d'un coup un regain d'intérêt, notamment celles 
qui sont en prise directe avec le numérique et en convergence entre elles, à savoir l'éducation aux médias, à l'information et à l'informatique. Elles en viennent à remanier les cartes des "disciplines » qui les hébergent, notamment les sciences de l'information-communication et les sciences de l'éducation, affectant la forme universitaire autant que la forme scolaire.

Donc l'injonction actuelle de créativité est à prendre avec précaution, notamment dans le cadre de l'école de la république héritée du XIX ${ }^{\mathrm{e}}$ siècle qui, entre-temps, a développé la forme scolaire aussi à des fins citoyennes et démocratiques. Elle relève toujours d'une commande industrielle, avec la promesse à venir d'emplois, mais elle est censée se couler dans une « forme » scolaire et universitaire pré-numérique qui lui résiste. Elle pose que tout individu, potentiellement, est créatif, tout le temps - ce qui à tout le moins est contre-intuitif avec la position antérieure, qui s'appuyait sur la mise en conformité et la stabilité de la reproduction. C'est une vision très productiviste, qui risque de produire des automatismes et non une nouvelle forme d'humanisme.

Le débat peut être posé en termes d'humanités numériques ${ }^{2}$ dans la mesure où il s'agit de dépasser la seule perspective productiviste et de préserver les acquis des Droits de l'homme en relation à l'école avec le passage aux technologies du numérique. Ces nouvelles littératies affectent en effet la vie privée (article 12), la liberté d'expression (article 19), l'éducation (article 26) et la participation (article 27) et sont affectées en retour et doivent être investies de sens pour être adoptées par les enseignants et les apprenants, à l'école comme à l'université.
Pour que la créativité ait sa place à l'école comme à l'université, dans une perspective humaniste et non pas seulement productiviste, il faut donc l'apprécier à la fois sous l'angle de la théorie de l'information-communication (quels modes de communication ? quelles valeurs de l'information? quelles postures pédagogiques? Comment articuler les nouveaux usages littéraciques aux apprentissages ?) mais aussi de la sociologie de l'action en éducation, sous l'angle des pratiques professionnelles et des politiques publiques (quels modes d'accès et de formation? Avec quels supports, espaces, compétences ?). En sachant que des résistances vont se présenter : la « forme » scolaire pré-numérique et sa capacité à évoluer, voire à se dé-former, le périmètre de l'éducation et sa capacité à performer efficacement avec l'adjonction de nouvelles littératies et enfin l'extension de l'éducation aux médias aux cultures de l'information et le risque de découplage entre médiatique, informatique et numérique ${ }^{3}$.

\section{La créativité : explorer les possibles ?}

Dans les sciences humaines et sociales, les définitions de la créativité foisonnent, qui l'inscrivent d'emblée dans la complexité. En psychologie différentielle, Todd Lubart propose une approche par compétence : «La créativité est la capacité à réaliser une production qui soit à la fois nouvelle et adaptée au contexte dans lequel elle se manifeste ${ }^{4}$. » Les sciences cognitives l'associent à un processus mental qui vise la résolution de problèmes par l'exploration des possibles : il s'agit d'inventer une solution à une question dont le résultat n'est pas connu, pas plus que la méthode pour y arriver. Le résultat escompté 
passe par toute une série d'hypothèses et de remaniements et se traduit par une réalisation concrète. Toutefois si celle-ci a l'air objective, elle n'en dépend pas moins du talent et du savoirfaire d'un individu, qui a son style créatif. Face à la possibilité de créer à l'infini (puisque tout peut être remanié), se trouve la relativité des situations humaines ${ }^{5}$.

En psychologie de l'enfance, Donald Winnicott associe lui aussi la créativité au faire, mais à partir de l'être, même si l'impulsion créative peut rester en repos. Ce n'est pas une réaction à l'environnement mais une action, au niveau de la vie quotidienne, qui relève du savoir-être et il l'associe au sentiment de soi plus encore qu'à la réalisation d'un objet. D'où son intérêt pour l'enfance, moment où se constituent l'omnipotence du regard et de l'action ainsi que la réalisation de la résistance de la réalité pour susciter l'élan créateur, en tenant compte de l'influence du milieu sur l'individu. L'apprentissage se fait par des «phénomènes transitionnels », qu'il relie à la croyance et à la culture en ce qu'ils créent chez l'adulte des espaces mentaux à la fois subjectifs et objectifs. C'est particulièrement le cas du jeu comme « espace potentiel » selon Winnicott, « aire transitionnelle d'expérience à laquelle contribuent simultanément la réalité intérieure et la vie extérieure ». Cette aire apparaît comme « un lieu de repos pour l'individu engagé dans cette tâche interminable qui consiste à maintenir à la fois séparées et reliées l'une à l'autre réalité intérieure et réalité extérieure ${ }^{6}$.»

En psycholgie du travail, chez l'adulte, Abraham Maslow conforte cette position. Il s'intéresse aux états de conscience exceptionnels, pour com- prendre la motivation et l'accomplissement de soi qui se manifestent selon lui par la créativité et l'engagement. Il considère que ce sont des besoins d'ordre supérieur (par contraste avec les besoins d'ordre physiologique de survie et sécurité) ; ils sont associés à l'estime de soi et au sentiment d'appartenance et d'attachement et sont eux aussi spécifiques à une culture et à ses croyances $^{7}$.

La psychologie humaniste de Maslow peut se rapprocher de la philosophie de Gilles Deleuze qui développe la notion de devenir dans Mille Plateaux ${ }^{8}$. Il considère le devenir comme «un processus du désir », avec des différentiels d'intensité qui font découvrir de nouveaux voisinages loin de l'imitation. Ces connexions inédites s'organisent dans un processus de déterritorialisation, qui ouvre la possibilité de produire du nouveau dans un acte créateur, loin du sens commun. Il rattache le devenir à l'affect, avec des intensités et des variations de degré qui permettent à l'individu d'éprouver une augmentation (ou une diminution) de sa puissance d'agir. Il l'inscrit en cohérence avec ses réflexions sur la coexistence du passé et du futur dans une esquive du présent, pour des besoins de simultanéité du vécu, car «le devenir ne supporte pas la séparation ni la distinction de l'avant et de l'après, du passé et du futur ${ }^{9}$. »

La définition de la créativité s'avère donc composite : elle n'est plus nécessairement connectée à une origine artistique et ne relève plus du territoire exclusif des artistes sui generis; elle est connotée à l'originalité dans les processus et à la résolution de problèmes, en lien avec l'agir, ce qui la rapproche d'un autre mot à la mode, 
« design »; elle parait avoir pour alliée la sérendipité, de l'ordre de l'inattendu et de l'inédit, ce qui la rapproche de la pensée latérale ou de la divergence ; elle semble relever d'une déterritorialisation et d'une délinéarisation temporelle proches de l'expérience actuelle de la mondialisation, qui implique une relation différente à soi et à l'autre, permettant des expériences de collaboration, y compris transfrontières et transmédias, autres notions souvent associées à la créativité de nos jours.

De fait, la créativité apparaît comme un processus agile. Elle ne se définit pas de manière stable; elle n'obéit pas à un canon; elle ne suit pas de stratégie spécifique. D'un point de vue pédagogique, la plupart des auteurs font valoir que chaque système scolaire peut favoriser ou tuer l'élan créateur de l'individu. Il faut donc créer les conditions de la créativité ... et espérer qu'au bout du processus, elle produise des individus en maîtrise des nouvelles littératies pour pouvoir jouer de leur réalité intérieure et des contraintes de la vie extérieure afin de trouver leur style créatif.

Le renouveau d'intérêt pour la créativité tient aux caractéristiques de l'informatique - qui sous-tendent le numérique - en ce qu'elle offre la possibilité de remanier tout contenu, indépendamment de son support d'origine : avec la pixellisation, toute information (textuelle, sonore, visuelle) devient sécable et malléable à volonté, y compris portable. Le numérique peut donc faciliter, voire décupler, la capacité de l'individu de créer à l'infini puisque tout document peut être remanié, mixé et morphé. Il permet ainsi d'explorer des possibles, de remanier des expé- riences, de produire des différentiels d'intensité. L'ordinateur devient l'interface de co-construction de la créativité et le support de nouvelles littératies qui exigent la maîtrise de la technologie et des savoir-faire propres aux cultures de l'information, matière première désormais indispensable à la production numérique. Il inscrit le savoir-devenir aux côtés du savoir-faire et du savoir-être, pour que l'individu puisse procéder à la mise à jour de soi, à l'exploration et à la réorientation de ses flux de possibles, à la modélisation ludique de ses lignes de vie ${ }^{10}$.

Le numérique est en outre lié aux affects et intrinsèquement médiatique. Il constitue à la fois une révolution de la communication par l'interactivité qui se manifeste sur les médias sociaux (Info-média) et une révolution de l'information qui se manifeste par la re-documentarisation du monde (Info-doc) et l'exploitation des données massives (Info-Data). Il envahit désormais l'école en joignant deux espaces traditionnellement disjoints, l'espace médiatique et l'espace scolaire, faisant de chaque apprenant un média potentiel, capable de créer ses contenus, de les diffuser et de réagir à ceux produits et diffusés par d'autres. Il met donc différentes « éducations à » en mouvement, notamment l'éducation aux médias, à l'information et à l'informatique.Il déplace du coup le monopole des pédagogies de la transmission pré-numériques et donne leur place légitime aux pédagogies du projet et de l'agir communicationnel, notamment l'éducation aux médias, qui a toujours prôné l'importance de la créativité. 


\section{Créativité et nouvelles littératies : changement de modèles}

\section{Modèles pré-numériques}

Quatre modèles discursifs sont en concurrence pour l'éducation aux médias pré-numériques : transmission, compétence, citoyenneté et créativité. Ils sont plus ou moins mis en œuvre dans les écoles et les pratiques pédagogiques, avec une distribution diverse en Europe et dans le monde, selon les situations nationales. Ces modèles tendent, avec des degrés d'intensité divers, à se focaliser sur 3 compétences (les 3C) : Compréhension,Critique (Pensée) et Créativité ${ }^{11}$.

Le modèle de transmission s'appuie sur la compréhension et vise à comprendre les médias et à les utiliser efficacement pour transmettre des informations. Il est basé sur une vue de l'enseignement liée au patrimoine et à la culture avec des méthodes et des ressources qui sont relativement limitées et basées sur l'écrit et le texte. Le professeur est au centre et les apprenants doivent apprendre par cœur, avec des exercices pré-établis et des tâches à reproduire.

Le modèle de compétence est présenté comme une alternative au modèle de transmission. Il vise à placer les apprenants au centre du processus, pour leur permettre d'être conscients des usages médiatiques et de leurs effets. Les activités ont tendance à vérifier les niveaux de conscience et la pensée critique avec un mélange d'exercices et de tâches qui produisent des attitudes réflexives et des aptitudes spécifiques (savoir-être et savoirfaire).
Le modèle de citoyenneté s'appuie sur la critique et tend à présenter les médias par rapport à la sphère publique et la création d'une opinion éclairée. Il essaye de favoriser la participation et l'engagement chez les jeunes, souvent en proposant des activités qui ont lieu à l'extérieur du cadre scolaire. Enseignants et apprenants travaillent ensemble avec d'autres acteurs pour s'engager dans des événements médiatiques (la Semaine de la Presse, la fête de l'Internet...) qui sont des événements d'apprentissage.

Le modèle de créativité propose une approche de terrain par l'utilisation directe des médias eux-mêmes et tend à déplacer la centralité du texte au profit de l'image et du son, voire du multimédia. Il pose en principe que la production de médias produit de la pensée critique et une meilleure compréhension des processus de communication et d'information. L'enseignant facilite l'appropriation des pratiques médiatiques pour susciter l'engagement des apprenants, particulièrement avec les outils du Web 2.0. Il place le projet au centre de l'apprentissage, qu'il soit individuel ou collectif. Les 3C (Compréhension, Critique et Créativité) s'enrichissent d'autres compétences extra-scolaires pour donner $7 \mathrm{C}$ : Citoyenneté, Consommation, Communication, Conflit (résolution) ${ }^{12}$.

Les modèles de transmission et de compétences ont rivalisé entre eux au cours des dernières décennies. Ils sont très présents dans les écoles car ils ne remettent pas en cause fondamentalement la forme scolaire, dans la mesure où ils suscitent de la connaissance et produisent des attitudes et des valeurs. Le modèle de créativité, basé sur des pédagogies actives tout comme 
le modèle de citoyenneté, peine à établir sa légitimité dans le système scolaire mais est très présent hors l'école, dans des lieux où l'accès à la technologie est facilité (fablabs, forges, ateliers de création ...).

\section{Modèles numériques et translittératie}

Les modèles numériques ont le potentiel d'inverser les modèles pré-numériques, car ils tendent à favoriser la créativité par une approche de terrain et par un brouillage de la division entre activités scolaires et péri-scolaires. La créativité y occupe une place centrale, avec un modèle discursif supplémentaire, le modèle de participation, qui renouvelle le modèle de citoyenneté.

Le modèle de participation privilégie la coopération et l'engagement, d'autant que les médias et les technologies sont devenus faciles à mettre en œuvre, avec des coûts réduits et des fonctionnalités accrues (mémoire, édition, radiodiffusion, micro-blogging...). Il s'appuie sur les médias sociaux et sur le co-design, la co-conception et la co-construction de connaissances.

Cette inversion est soutenue par un changement de paradigme, en raison de la transformation de la notion d' « information ». Il exige de revoir les territoires des cultures de l'information, à mesure que la computation (Info-data), la communication (Info-médias) et la documentation (Info-doc) convergent autour des significations feuilletées du terme « translittératie » défini comme :

«1. La capacité de s'approprier le multimédia avec des compétences de lecture, écriture et calcul applicables à tous les outils disponibles (du texte à l'image, du livre au wiki) ;

2. La capacité de naviguer à travers des domaines multiples qui incluent l'aptitude à rechercher, évaluer, tester, valider et modifier l'information selon ses contextes pertinents d'utilisation (code, actualité et document) ${ }^{13}$.»

Dans cette perspective augmentée, les compétences pré-numériques, les $7 \mathrm{C}$, restent valables, mais s'enrichissent de la convergence des trois cultures de l'information: Info-Data (informatique), Info-Media (communication) et InfoDocument (information) (voir Illustration $\mathrm{n}^{\circ} 1$ ).

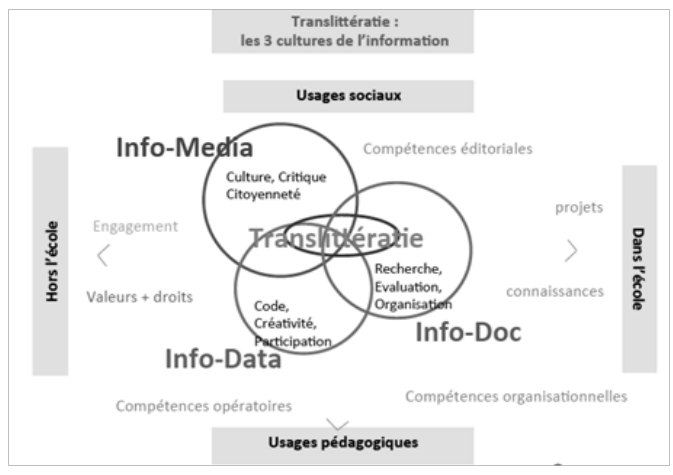

Illustration $n^{\circ} 1$ : Translittératie: les trois cultures de l'information (source : ANR TRANSLIT)

Les compétences numériques exigées pour la translittératie sont opérationnelles (coder, calculer, traiter), éditoriales (trier, évaluer, publier) et organisationnelles (sourcer, rechercher, naviguer $)^{14}$. Quoiqu'elles puissent être maîtrisées par un individu, elles relèvent davantage de « compétences distribuées », pour prendre en compte le modèle participatif, où les personnes travaillent en groupes autour de projets où elles fonctionnent tant en mode bricolage (Do It Yourself ou DIY) qu'en mode partage (Share It With Others ou 
SIWO). Il n'est plus indispensable à une personne de tout maîtriser mais davantage de s'insérer dans un assemblage de compétences apportées par d'autres, qu'il est possible d'acquérir (ou pas) par le faire ensemble et avec - d'où l'importance des compétences non techniques sociales (soft skills) souvent associées de nos jours à la créativité, relevant du savoir-être et du savoir-devenir plus que du savoir-faire.

Ces compétences distribuées recombinent les caractéristiques des trois cultures de l'information et développent la créativité, comme phénomène distribué lui aussi. Elles y parviennent du fait de la capacité du numérique à remanier tout contenu, indépendamment de son support d'origine à laquelle s'ajoute la capacité des personnes à diffuser à l'infini ces contenus remaniés, du fait des valeurs de partage, d'interopérabilité et d'ouverture associées à l'informatique depuis ses origines. Ce processus promeut la réflexivité (suivre ses traces par le biais des données), la collaboration (mixer ses traces avec celles des autres) et la créativité (de l'apprentissage par imitation à l'apprentissage par simulation).

Ce processus alimente également la présence en ligne, éprouvée non plus comme une expérience de la distance mais comme un ressenti de la proximité. Elle est composée de dimensions différentes : la présence 'cognitive' définie comme « la façon dont les apprenants peuvent construire de la signification par une réflexion soutenue et un discours critique inscrit dans une communauté de recherche toujours en quête de sens »; la présence 'sociale' définie comme "la capacité des participants à une communauté de recherche de se projeter socialement et émotionnellement, comme des personnes 'réelles' (avec la totalité de leur personnalité) à travers le moyen de la communication utilisé15. » Ces deux dimensions sont importantes «pour assurer la pleine mâेtrise de la présence 'designée', définie comme la façon dont les apprenants ont conscience des contraintes et des potentialités intégrées dans le moyen de communication utilisé. Ceci implique la compréhension des finalités (souvent commerciales) qui régissent les outils d'information, curation et création à disposition, ce qui peut avoir des incidences sur la performance personnelle ${ }^{16}$. »

La translittératie est donc une éducation aux médias et à l'information augmentée par le numérique qui permet aux apprenants de mobiliser leurs propres scénarios cognitifs (comme unités de sens et unités de prise de décision) et de faire appel à leurs expériences pour adapter et contrôler leur propre performance en ligne et leur interaction avec les autres. Ils doivent faire appel à leurs représentations des autorités qui contrôlent la prestation des outils numériques pour les adapter à leurs propres besoins si nécessaire. En ce sens, la translittératie doit intégrer des bases informatiques (variables, algorithmes, probabilités...) pour que le code ne soit pas une suite opaque de chiffres, mais elle doit aussi inclure la pensée critique propre à l'éducation aux médias pour évaluer la façon dont les plateformes commerciales ou pédagogiques manipulent les contenus et affectent leurs performances.

\section{Translittératie : au carrefour de la médiation et de la médiatisation}

De telles avancées en sciences sociales et 
cognitives comme en technologie doivent être intégrées dans le système scolaire et la formation des enseignants. Cette formation doit se déplacer du modèle de transmission encore dominant aux modèles de créativité et de participation. Elles suggèrent une forte focalisation sur la translittératie et les retombées en e-présence pour les apprenants, quel que soit leur âge. La modélisation des processus d'apprentissage par la translittératie ne suffit pas sans une compréhension des inter-relations entre la médiation numérique et la médiation pédagogique, pour faire évoluer la forme scolaire et universitaire.

\section{La médiation numérique : répertoire des e-stratégies et humanités créatives}

Quand le numérique et la cognition s'appuient réciproquement par le biais des affordances technologiques, tout un répertoire de e-stratégies vient conforter la e-présence. Les fonctionnalités des plateformes numériques et de certains logiciels reproduisent fortement des stratégies cognitives du cerveau, ce qui explique qu'elles donnent le sentiment d'être intuitives et faciles d'usage, avec une ergonomie robuste et un design attractif. Elles recoupent également les besoins cognitifs du savoir-devenir et de la translittératie qui s'appuient sur l'ordinateur comme interface de créativité.

Henry Jenkins et ses collègues du MIT ont décrit un certain nombre de ces stratégies en ligne, observées dans les classes :

- Jeu (game) : aide à la résolution de problèmes, sans prise de risques,

- Simulation (simulation) : permet de tester des modèles dynamiques de processus applicables au monde réel,

- Agrégation de contenus (content-aggregation) : encourage l'expression d'identités alternatives pour la réorientation des choix,

- Échantillonnage (sampling) : expérimente avec le remixage des produits médiatiques pour une meilleure compréhension de la culture et la mise à jour de soi,

- Multi-modalisation (multitasking) : aide à l'interaction entre divers médias et diverses options au sein d'un même support,

- Mise en commun de ressources (pooling) : contribue à l'intelligence distribuée par agrégats de savoirs partagés (Share It With Others),

- Navigation transmédias (transmedia navigation) : favorise le contrôle sur l'information qui est accessible dans le domaine public et la création de nouveaux contenus,

- Réseautage (networking) : facilite la recherche et la distribution de l'information ainsi que l'engagement citoyen,

- Coordination pair à pair (peer to peer coordination) : peut produire de la négociation entre diverses communautés en ligne et hors ligne pour trouver des procédés alternatifs et générer des solutions innovantes ${ }^{17}$.

Ce répertoire de e-stratégies, fondé sur des compétences distribuées, éclaire d'une lumière précise la translittératie comme une éducation aux médias et à l'information augmentée par l'informatique. Il affecte durablement la perception et la compréhension de la connaissance elle-même, et par contrecoup de la créativité.

Outre le répertoire de e-stratégies, la convergence entre informatique, information et médias a produit des fonctionnalités technologiques et cogni- 
tives spécifiques qui augmentent les humanités et peuvent produire une médiation numérique à l'école. Ces fonctionnalités se retrouvent dans des logiciels, pour la plupart gratuits, qui permettent quatre principaux modes de représentations nouvelles des savoirs, notamment de type LSHS :

- le traitement du langage naturel (par exemple Alceste),

- l'information géographique et le positionnement spatio-temporel (par exemple, Neatline),

- la visualisation de données (par exemple, Tableau Public),

- l'analyse des réseaux sociaux (par exemple, Gephi).

Elles se placent résolument du côté des modèles de créativité et de participation, puisqu'elles peuvent même aider à visualiser cette participation par des cartographies, des analyses d'apprentissage profond (learning analytics), etc.

Ces fonctionnalités et ces modes de représentation avec leurs logiciels d'exploration de données attenants démystifient les cultures de l'information: les données ne sont plus rares et secrètes mais abondantes et révélatrices de relations invisibles à l'œil nu. Elles risquent de rester lettre morte si elles ne sont pas explorées dynamiquement et démocratiquement, ce qui implique qu'elles fassent partie du bagage donné à tout un chacun dès l'école primaire et jusqu'à l'enseignement supérieur, c'est-à-dire une connaissance non experte (soft skills) mais toutefois raisonnée et critique du numérique.

Elles permettent aux humanités numériques de devenir pleinement des humanités créatives, c'est-à-dire d'opérer tout à la fois l'articulation des structures cognitives, des modes de représentation des savoirs et des cadres d'action socialisés. Elles ont un impact sur les communautés de sens et de pratique, sur les interfaces homme-machine et les productions, avec des gains en engagement et en motivation. À terme, elles ne peuvent qu'opérer une modification profonde de notre façon de percevoir le savoir et de construire la connaissance (modèles ouverts, co-construction, co-design...). La translittératie, comme éducation aux médias et à l'information augmentée par l'informatique, apporte une dimension critique à la créativité, qui n'est pas nécessairement présente dans la computation en tant que telle, avec ses propres contraintes et finalités. Les humanités créatives peuvent ainsi rester sous le contrôle de l'humanisme, et être éthiques dans leur design, y compris leur design pédagogique.

Les humanités créatives peuvent alors contribuer à la constitution des nouvelles industries du XXI siècle, notamment les industries dites " créatives » (musique, design, cinéma, architecture, jeux...) par contraste avec les industries culturelles pré-numériques. Le terme fait l'objet de tensions en ce qui concerne son périmètre, entre une définition étroite qui l'associe aux filières des arts appliqués et au design et une définition large qui recoupe les industries culturelles prénumériques (cinéma, jeux, film, musique...). Elle pointe vers des modes de financement et des pratiques numériques maîtrisées par l'usager et non par le mécénat public ou privé car « la diversité des modes de financement et des interactions entre secteurs de production, distribution et consommation impliqués suggère qu'à bien des égards, c'est la notion de participation - individuelle ou collaborative - qui est au cour 
des enjeux des industries créatives et qui les différencie des industries culturelles. Cela tient aux deux versants des biens culturels à l'ère numérique, le relationnel et l'expérientiel, fortement imprégnés par la culture des réseaux et par une représentation de la culture comme 'réseau cognitif distribué' selon Merlin Donald ${ }^{18}$. »

Adossées aux humanités créatives, les industries créatives peuvent être une façon d'explorer ce que James Lull évoque comme la possibilité de créer des « supercultures personnelles ${ }^{19}$ » (personal supercultures). Ulrich Beck fait référence à une collectivité paradoxale en parlant d' « individualisation réciproque ${ }^{20} \gg$ (reciprocal individualization) pour décrire ces nouvelles transitions sociales et culturelles tandis que Barry Wellman évoque des « communautés personnelles ${ }^{21} »$ (personal communities). Au sein de la translittératie, il s'agit à tout le moins de former des individus connectés, à compétences distribuées.

\section{La médiation pédagogique et la médiatisation : l'étayage cognitif}

La mise en œuvre de la créativité et de la participation à l'école comme à l'université ne peut se faire sans pédagogies innovantes, telles que celles visibles dans les MOOC (cours massivement ouverts en ligne), avec leurs divers styles d'apprentissages en lien avec les médias, comme le démontre le « modèle du cube pédagogique d'apprentissage » (learning cube pedagogical model) de Nishikant Sunwalkar, appliqué aux MOOC $^{22}$. Ce modèle adaptatif peut être augmenté en y ajoutant le répertoire de e-stratégies et les $7 \mathrm{C}$ de l'éducation aux médias et à l'information (Illustration $n^{\circ} 2$ ).

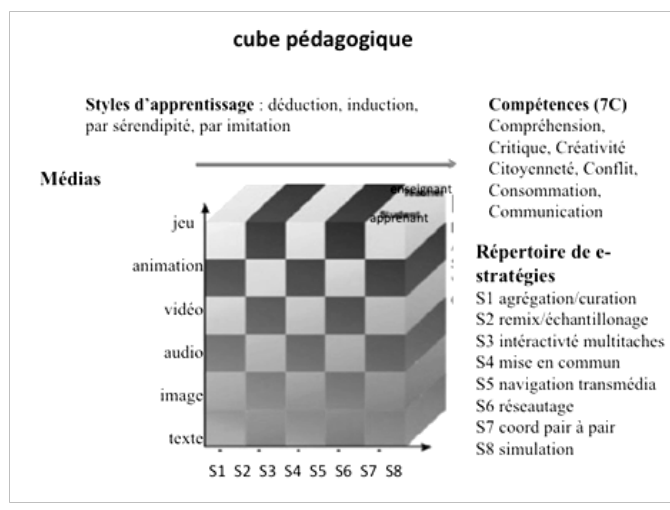

Illustration $n^{\circ} 2$ : Le cube pédagogique d'apprentissage augmenté par la translittératie (projet ECO)

Les MOOC du projet européen ECO favorisent ce modèle, d'autant que ce sont des sMOOC (SocialMOOCs), car leur design pédagogique se fonde sur les apports des réseaux sociaux et des communautés de pratique, notamment autour de l'éducation aux médias et à l'information, de la créativité et des usages participatifs du Web 2.023 (illustration $\mathrm{n}^{\circ} 3$ ).

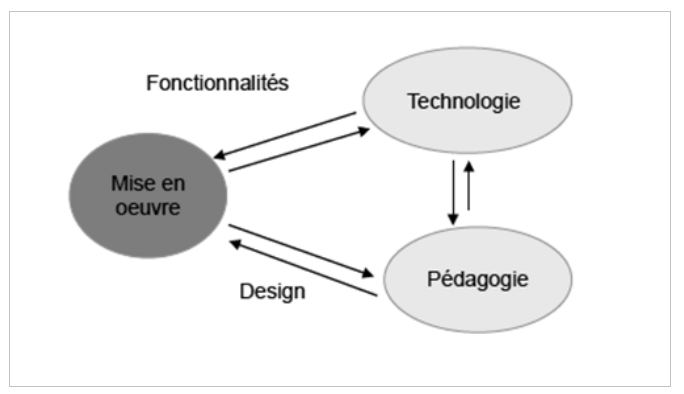

Illustration $n^{\circ} 3$ : Design pédagogique et technologique (projet ECO) 
Dans ce contexte, la médiation technologique ne peut se passer de la médiation pédagogique, pour être au plus près des besoins cognitifs des apprenants et susciter leurs styles créatifs propres. De fait elles se renforcent réciproquement : la médiation pédagogique offre un cadre d'action que le numérique tend à augmenter également, comme en témoigne l'adjonction systématique du suffixe «-tion » à de nombreux termes pré-numériques. Ce suffixe met le pointeur sur les processus, les usages et les pratiques. Il s'applique à toutes sortes d'activités translittéraciques et aux compétences distribuées : opération devient opérationnalisation, édition devient éditorialisation, etc. Les cultures de l'information semblent se redoubler, chaque processus pré-numérique trouvant une addition numérique : la médiation avec la médiatisation, le document avec la documentarisation, l'information avec l'informatisation ${ }^{24}$. De même pour la pédagogie qui devient pédagogisation à mesure que les pratiques numériques et la circulation sur les réseaux produisent une connaissance de plus en plus fine des processus d'apprentissage.
La médiation pédagogique s'articule avec les fonctionnalités et les modes de représentations adossés aux logiciels des humanités créatives, comme le traitement du langage naturel, l'information géographique et le positionnement spatiotemporel, la visualisation de données et l'analyse des réseaux sociaux. Des cadres d'action socialisés disjoints dans le pré-numérique peuvent alors se rejoindre. Cette articulation a un impact sur le design des interfaces, sur les outils et les productions selon l'espace et le temps alloué aux communautés de pratique. Cette articulation a également un impact sur les pédagogies numériques, particulièrement celles qui se fondent sur le projet de l'apprenant et utilisent l'étayage cognitif ${ }^{25}$ (Illustration $n^{\circ} 4$ ).

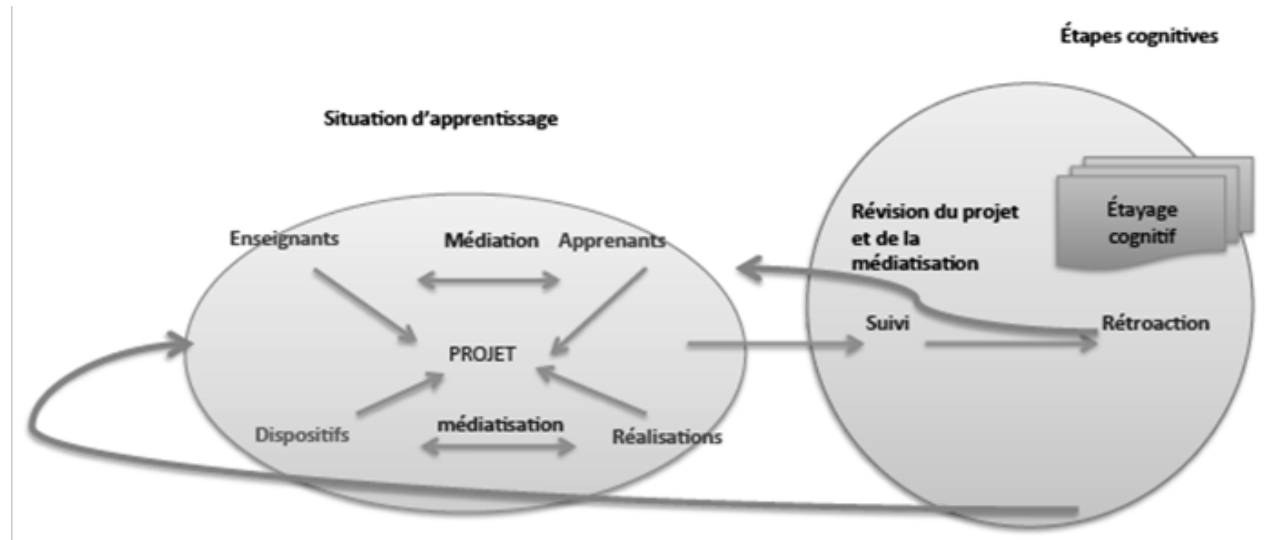

Illustration $n^{\circ} 4$ : Etayage cognitif (mis en œuvre dans le master AIGEME et le MOOC «DIY EMI ») 
La médiation pédagogique nécessite quelques conditions minimales d'apprentissage, qui impliquent la médiation des enseignants, des tuteurs et autres personnes ressources dont le statut est lui aussi modifié par le numérique. Elle incorpore aussi plus visiblement des processus cognitifs comme la rétroaction (feedback), la révision, qui rendent explicite la grammaire des usages par le biais de l'étayage cognitif. Celui-ci correspond au processus de créativité par lequel la résolution de problèmes se fait par l'exploration des possibles : il suggère toute une série d'hypothèses et de remaniements pour aboutir à une réalisation concrète. Appliqué à l'élaboration d'un projet, l'étayage cognitif permet de repérer les différentes étapes successives de modélisa- tion, révision et rétroaction, - fournies par la réflexivité mais aussi par la discussion avec les pairs et les experts ${ }^{26}$ (Illustration $n^{\circ} 5$ ).

L'étayage permet de valider certains outils et étapes, en les révisant constamment par rétroaction, mais aussi par activation des e-stratégies que sont la navigation, la mise en commun de ressources, l'échantillonage et le mixage. Ce montage complexe est confirmé par l'observation des pratiques chez les jeunes, telle qu'opérée par l'ANR TRANSLIT. Trois facteurs apparaissent comme bénéfiques pour une intégration réussie de la translittératie dans les enseignements : l'actualisation des stratégies individuelles et collectives, les compétences distribuées organisées

Illustration $n^{\circ} 5$ : Etayage cognitif (mis en œuvre dans le master AIGEME et le MOOC «DIY EMI »)

\begin{tabular}{|c|c|c|c|c|c|}
\hline Étayage au début du processus & & & & & \\
\hline Niveau en début de projet $=\mathrm{E}$ & & & & & \\
\hline $\mathrm{E}=\mathrm{Embryonnaire}$ & & & & & E \\
\hline$D=$ Développé de façon rudimenta & & & & D & \\
\hline $\mathrm{C}=$ Compréhensible mais avec trous, écarts & & & C & & \\
\hline B= Bien balisé, en bonne voie & & B & & & \\
\hline $\mathrm{A}=\mathrm{Acceptable}$, abouti au maximum & A & 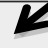 & & & \\
\hline & A & B & C & D & $\mathbf{E}$ \\
\hline -Étape 1 & & & & & E \\
\hline -Étape 2 & & & & & E \\
\hline -Étape n & & & & & $E$ \\
\hline
\end{tabular}

\section{Etayage en fin de processus}

\begin{tabular}{|l|l|}
\hline Niveau en fin de projet = A & \\
\hline A= Acceptable, abouti au maximum & A \\
\hline & A \\
\hline -Étape 1 & A \\
\hline -Étape 2 & A \\
\hline -Étape $\mathrm{n}$ & A \\
\hline
\end{tabular}


autour d'outils et dispositifs socio-technologiques et la médiation pédagogique organisée autour d'étayages cognitifs rendus explicites ${ }^{27}$.

\section{Implications pour les sciences humaines et sociales : vers la nécessaire transformation transdisciplinaire des LSHS}

Pour intégrer ces modèles de créativité et de participation à la translittératie, comme nouvelle pratique de l'écrit, du lu et du calculé, qui articule une technologie, la connaissance de celle-ci et des savoir-faire et des savoir-être particuliers (dont la présence en ligne), il faut envisager de nouveaux développements disciplinaires, qui réorganisent le socle des connaissances du XXI ${ }^{\mathrm{e}}$ siècle.

Ils doivent faire l'objet de politiques publiques fondés sur les résultats de la recherche, autour des trois cultures de l'information. Les sciences dites « dures » ont commencé cette mutation et se réorganisent autour de l'information par le biais de l'alliance STEM (Science, Technologie, Engineering, Math), appuyées par la National Science Foundation aux États-Unis. Percolant en réseau vers d'autres pays et régions du monde, elle est soutenue en Europe par le réseau European Schoolnet et s'organise autour d'activités menées en mode projet, autour de la robotique (capteurs, senseurs...), ce qui permet de faire converger les différentes sciences avec divers styles d'apprentissage.

Les sciences humaines et sociales - dont les « softskills » sont pourtant au centre des transformations actuelles - peinent à opérer cette transformation, alors qu'elles ont elles aussi l'information au cœur de leur écosytème, surtout si sont prises en compte les nouvelles formes de représentation des savoirs LSHS (traitement du langage naturel, géolocalisation, visualisation de données et des réseaux sociaux). Il leur faut, elles aussi, s'organiser en alliance, une alliance SMILE (Synergies for Media and Information Literacy in Education), avec les compétences distribuées de la translittératie comme pivot. Elles doivent soutenir l'extension des humanités créatives pour s'assurer que des individus engagés participent aux industries créatives de demain, tout en restant critiques et sensibles aux droits de l'homme ${ }^{28}$.

\section{Quelques principes}

- Un cadre conceptuel autour de la translittératie, qui favorise les compétences distribuées (opératoires, éditoriales et organisationnelles).

- Une pédagogie du projet, avec étayage cognitif, pour intégrer les e-répertoires de stratégies.

- Une formation des enseignants à partir d'un nouveau socle commun de compétences, de connaissances et de culture qui joigne disciplines universitaires, dispositifs socio-technologiques, usages finalisés et projets de l'apprenant, sans rupture entre l'informatique et les cultures de l'information (voir illustration $\mathrm{n}^{\circ} 6$ ).

Le but de cette formation basée sur le socle plutôt que sur le programme est de former des apprenants qui sont les constructeurs actifs de ce qu'ils apprennent (par curation, agrégation de contenus, mise en commun des connaissances...). Ce processus devrait créer des individus autonomes avec la capacité de gérer leur présence 
ainsi que leur répertoire de stratégies, pour être actifs sur les réseaux sociaux, engagés dans le design de la démocratie et la promotion des droits de l'homme.

Cette articulation entre la transmission du contenu, attachée aux disciplines universitaires pré-numériques et l'acquisition de processus transversaux d'apprentissage eux-mêmes associés à des outils socio-techniques, doit être inscrite dans les plans de formation. C'est sans doute le maillon faible des systèmes actuels, qui rend l'intégration de la translittératie difficile, dans la mesure où la formation des enseignants tend à se produire dans des disciplines organisées en silos, sans passerelles entre elles et avec les « éducations à ».

Deux types d'implications en découlent : l'importance de construire un domaine public en ligne pour disséminer la culture et les connaissances et la nécessité de reconnaître les pédagogies innovantes, comme les MOOC, et de valoriser leurs modèles d'apprentissages différenciés.

IIIustration $n^{\circ} 6$ : matrice de formation SMILE (modèle master AIGEME)

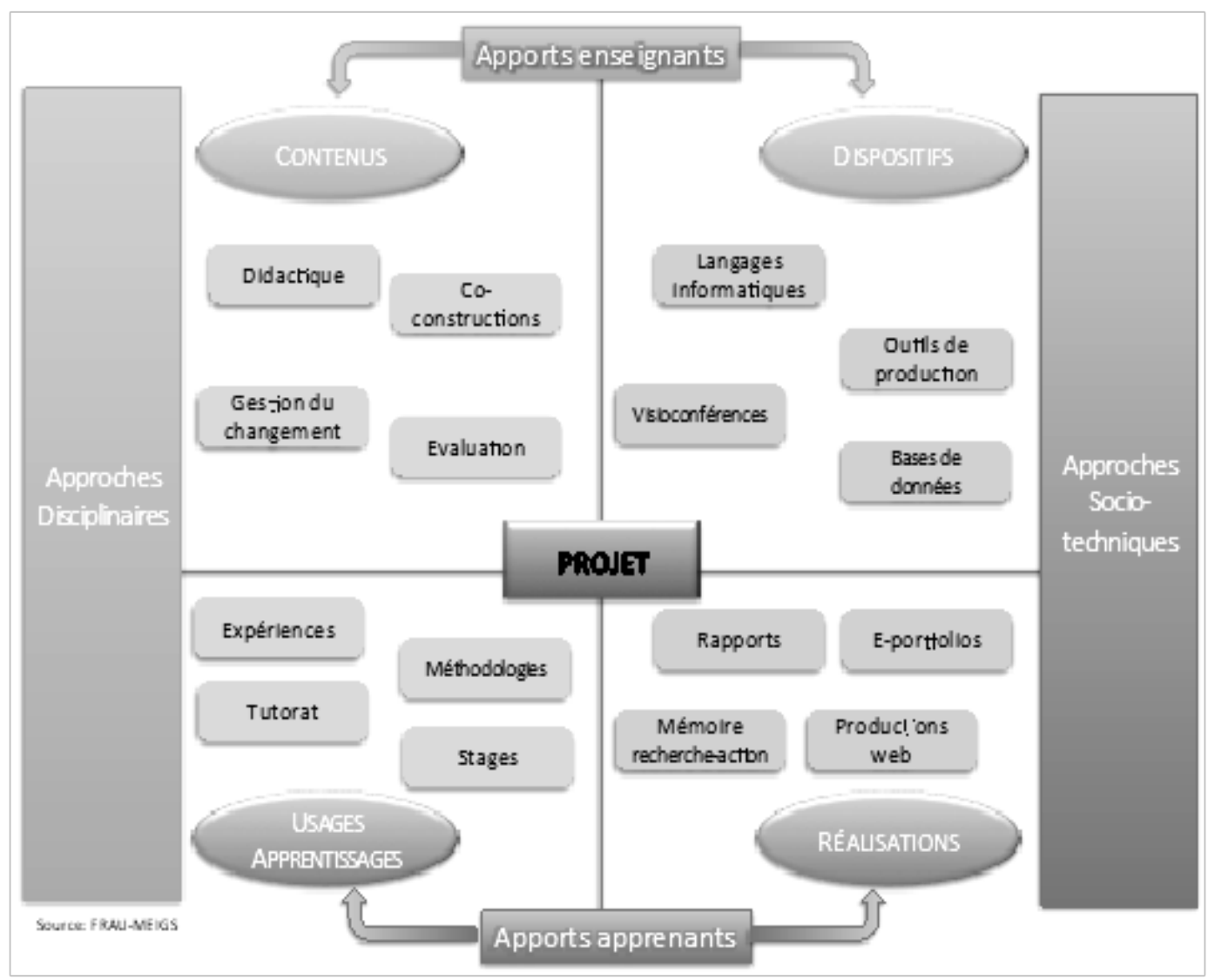




\section{Mise en ouvre : alliance SMILE}

Pour mettre en œuvre cette vision partagée, le service public de l'enseignement doit prévoir un financement, des ressources, des formations et des évaluations spécifiques. Cela implique aussi une modification profonde de la forme scolaire, qu'il faut équiper avec des tiers lieux (bibliothèques, learning centers, fabeliers...) et de quarts lieux (des plates-formes en ligne). La rénovation doit être appuyée par la continuité pédagogique dans et hors l'école, de la maternelle à l'université.

Le périmètre de la translittératie, entre infomédia, infodocumentation et infodata, doit être défini plus précisément. La responsabilité diffuse de cette « éducation à », qui n'est pas vraiment attribuée à un enseignement spécifique ou à une équipe désignée l'affaiblit. La position marginale des méthologies et pédagogies transversales doit être revalorisée, afin de susciter des vocations dans les humanités créatives et avoir accès aux nouveaux métiers qu'elles promettent ${ }^{29}$.

La construction de communautés de sens et de pratique via des pédagogies participatives peut être un moyen pour augmenter progressivement la translittératie. L'apprentissage en ligne et l'accès aux Ressources Éducatives Ouvertes (REL) via les MOOC et le développement du champ de la cognition et de la socio-construction sont des forces sur lesquelles il est possible de compter. L'enseignement aux médias par les médias peut faire partie d'une stratégie à long terme pour la formation continue et tout au long de la vie.

La translittératie peut mener au pouvoir d'agir et au savoir-devenir si elle est mise dans un cadre de bonne gouvernance où les avantages des nouveaux modes cognitifs d'apprentissage sont partagés, centrés sur les apprenants et pas simplement induits par la technologie. La translittératie comme éducation aux médias et à l'information augmentée par le numérique détient le potentiel de réduire les disjonctions entre les divers types de médias (papier, analogique, numérique), les conflits entre culture de l'élite et culture populaire, entre les systèmes propriétaires et non propriétaires, etc. Elle propose un scénario pour le développement numérique durable, particulièrement avec ses ressources partagées et ses initiatives open source.

La translittératie doit être accompagnée par une réflexion éthique sur l'internet des sujets en lien avec l'internet des objets, rattachée aux droits de l'homme, afin d'apprivoiser et civiliser le numérique $^{30}$. Cela suppose de nouvelles dynamiques institutionnelles, qui accompagnent les modifications de la forme scolaire et de la forme universitaire, au risque que celles-ci soient marginalisées et remplacées par les plateformes numériques en peine de recrutement des nouveaux travailleurs $\mathrm{du} \mathrm{XXI}^{\mathrm{e}}$ siècle (forgers, start-upers, makers, hackers...). Ce processus et ces conditions de mise en œuvre, pour passer à l'échelle nationale, doivent faire l'objet de politiques publiques, fondées sur les résultats de la recherche ${ }^{31}$. Celle-ci suggère de s'appuyer fortement sur les dimensions matérielles et symboliques de l'information et de la communication. Mais l'optique des sciences de l'informationcommunication en France doit elle-même se modifier pour incorporer davantage la complexité de l'écosytème numérique, le multi-transmédia et 
la translittératie. La diversité ethnographique des usages et pratiques formelles et informelles, la théorisation des relations de pouvoir et de savoir à l'interface des e-répertoires de stratégies et des cadres d'action numérique doivent impérativement être pensées et portées par les LSHS, dans la perspective d'une alliance SMILE.
$N$

$\mathrm{N} \cdot \mathrm{O} \cdot \mathrm{T} \cdot \mathrm{E} \cdot \mathrm{S}$

1. W. Benjamin, L'Euvre d'art à l'époque de sa reproductibilité technique (1939), traduit par F.Joly (Paris : Payot, 2013).

2. M. Doueihi, La grande conversion numérique (Paris: Seuil, 2008); M. Doueihi, Pour un humanisme numérique (Paris: Seuil, 2011) ; M. K. Gold, Debates in the Digital Humanities (Minneapolis: University of Minnesota Press, 2012).

3. Cette analyse se fonde sur des modélisations et mises en œuvre effectuées au cours de différentes recherches menées au sein de la Chaire Unesco « Savoir Devenir », sous ma responsabilité, entre 2013 et 2017: l'ANR TRANSLIT sur la convergence entre éducation aux médias, à l'information et à l'informatique (http:// translit.univ-paris3.fr/), le projet européen ECO (Communication, Mobile Open learning) visant à développer des MOOC destinées aux compétences numériques (www.ecolearning.eu), dont le MOOC «Pas à Pas » (https://mooc-francophone.com/cours/ mooc-pas-a-pas/) et le MOOC « DIY EMI », financé par l'ANR (https://mooc-francophone.com/cours/ mooc-diy-education-aux-medias-et-a-linformation/). Elle repose également sur des observations portées pendant dix ans sur les productions de e-portfolios du master pro AIGEME que j'ai créé et dirigé de 2005 à 2015 à l'université Sorbonne Nouvelle (www.univparis3.fr/master-2-aigeme).

4. T. Lubart, Psychologie de la créativité (Paris : Armand Colin, 2003).

5. M. Donald, Origins of the Mind:Three Stages in the Evolution of Culture and Cognition (Cambridge: Harvard UP, 1991); D. Frau-Meigs, Penser la société de l'écran. Dispositifs et usages (Paris: Presses de la Sorbonne Nouvelle, 2011).

6. D. W. Winnicott, Playing and Reality (London : 
Routledge, 1971).

7. A. Maslow, "A theory of human motivation", Psychological Review, 50 (1943) : 370-396. (trad. fr : 1964).

8. G. Deleuze et F. Guattari, Mille Plateaux (Paris : Éditions de Minuit, 1980).

9. G. Deleuze, Logique du sens (Paris : Éditions de Minuit, 1969).

10. C'est précisément le projet développé par la Chaire UNESCO « Savoir Devenir dans le développement numérique durable : maîtriser les cultures de l'information » http://savoirdevenir.net/chaireunesco/ objectifs-missions/concepts-cles/

11. C. Bazalgette, Teaching Media in PrimarySchools (London : Sage, 2010) ; voir aussi D. Frau-Meigs, Socialisation des jeunes et éducation aux médias (Toulouse : Eres, 2011).

12. D. Frau-Meigs, " Augmented Media and Information Literacy (MIL): How can MIL harness the affordances of digital information cultures? » R. Kupiainen and S. Kotilainen (eds). Media Education Futures (Goteborg: Clearinghouse, 2015).

13. D. Frau-Meigs, «Transliteracy as the new research horizon for media and information literacy $\gg$ Meduskestudije/Media Studies 36 (2012) : 16 ; Voir aussi E. Delamotte, V. Liquète et D. Frau-Meigs, « La translittératie dans les cultures de l'information: supports, contextes et modalités », Spirales, 53 (2013) : 145-156. 14. A. Serres, Dans le labyrinthe. Évaluer l'information sur internet (Caen: C\&F éditions, 2012) ; B. Drot-Delange et E. Bruillard, « Éducation aux TIC, cultures informatique et du numérique : quelques repères historiques », Études de Communication 38 (2012) : 69-80 ; voir aussi J. Maeda, Creative Code (New York: Thames and Hudson, 2004) et S. Vial, Court traité du design (Paris: PUF, 2010).

15. D.R. Garrison and T. Anderson, E-Learning in the $21^{\text {st }}$ century. A framework for research and practice
(London: Routledge, 2006) 28-29; D. Frau-Meigs \& A. Bossu, "Towards e-presence at distance as a way to reach and share e-quality: the case of the ECO sMOOCs » in C. Delgado Kloos, P. Jermann, M. Pérez-Sanagustín, D. Seaton, S. White (eds), Digital Education : Out to the World and Back to the Campus (Heidelberg : Springer, 2017).

16. D. Frau-Meigs, « Transliteracy: sense-makingmechanisms for establishing e-presence » U. Carlsson (ed) Media and Information Literacy and intercultural dialogue (Goteborg: Clearinghouse, 2013) 175-189. 17. Adapté de H. Jenkins et al. Confronting the Challenges of Participatory Culture. Media Education for the $21^{\text {st }}$ Century (Chicago: MacArthurFoundation, 2006). Voir d. boyd, It's complicated: The social lives of networkedteens (London/New Haven: Yale UniversityPress, 2014); voir aussi S. Livingstone \& J. Sefton-Green, The class. Living and learning in the digital age (New York: NYU Press, 2016).

18. D. Frau-Meigs, « industries créatives », D. FrauMeigs et A. Kiyindou (eds), La diversité culturelle à l'ère numérique : glossaire critique (Paris : La Documentation française, 2014) 132-34.

19. James Lull, Culture-on-Demand: Communication in a Crisis World (Oxford: Blackwell, 2008), notamment le chapire 3 « Programming our personnal supercultures ».

20.U. Beck, \&E. Beck-Gernsheim, Individualization: Institutionalized Individualism and its Social and Political Consequences (London: Sage, 2002).

21. B.Wellman, Networks In The Global Village: Life In Contemporary Communities (Boulder, CO: Westview Press, 1999).

22. N.Sonwalkar, «The First Adaptive MOOC: A Case Study on Pedagogy Framework and Scalable Cloud Architecture-Part I », MOOCS Forum, 2013, 24.

23. Le catalogue complet des MOOC du projet ECO est disponible à www.ecolearning.eu. 
24. M. Zacklad, « Documentarisation processes in Documents for Action (DofA): the status of annotations and associated cooperation technologies ", Computer Supported Cooperative Work 15 2-3 (June 2006): 205-228. https://www.academia.edu/4236806/ Documentarisation_processes_in_Documents_for Action_DofA the status_of_annotations_and_associated_cooperation technologies

25. J-P. Narcy Combes, Didactique des langues et TIC : vers une recherche-action responsable (Paris: Ophrys, 2005) ; N. Yelland and J. Masters, « Rethinking scaffolding in the information age » Computers and Education 48 (2007):362-382; H. Hartman, « Scaffolding and Cooperative Learning » Human Learning and Instruction (New York: City College, 2002).

26. Voir le MOOC ECO «DIY Éducation aux Médias et à l'Information » qui fonctionne sur la base de l'étayage cognitif, disponible https://hub5.ecolearning. eu/media/attachments/Le_MOOC_DIY_EMI.pdf 27.Voir rapport final de l'ANR TRANSLIT, disponible http://translit.univ-paris3.fr/

28. Comme le suggère la définition des villes créatives par l'UNESCO, disponible http://www.unesco. org/new/en/culture/themes/creativity/creative-citiesnetwork/about-creative-cities/

29. Voir l'évolution du référentiel national des métiers et certifications professionnelles, disponible www.rncp.cncp.gouv.fr/

30. Voir la recommandation du conseil de l'Europe sur l'Internet des Citoyens, Strasbourg, mars 2015, disponible https://www.coe.int/fr/web/culture-and-heritage/ recommendation-on-the-internet-of-citizens; voir aussi la recommandation sur les données massives, la littératie et la démocratie, Strasbourg, septembre 2017, disponible https://rm.coe.int/1680750d68

31. D. Frau-Meigs, I. Velez \&J.Flores, Public Policies in Media and Information Literacies in Europe: Cross Country Comparisons. Londres : Routledge, 2017.

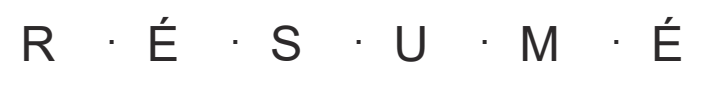

Autrefois, la créativité n'était pas une priorité pédagogique. Aujourd'hui, les enseignants et les artistes se réinventent « geeks » ou « designers » de contenus. De manière croissante, les modèles scientifiques sont en train de donner une nouvelle forme à la « créativité » à travers le Web, combinant « médiation » et « médiatisation » afin de construire un répertoire des « e-strategies » en « humanités créatives ». Ce renouvellement des études créatives modifie les Humanités classiques en les invitant à une forme de coopération interdisciplinaire. La translittératie définie comme la convergence de l'éducation aux médias, de l'éducation à l'information et de l'informatique rend possible l'autonomisation. Ce projet rend possible de nouveaux modes cognitifs d'apprentissage partagés, centrés sur l'apprenant, libérés de l'aspect technologique dans la perspective d'une alliance SMILE (Synergies for Media and Information Literacies) élaborée dans un cadre européen de gouvernance multipartite lui-même rendu nécessaire par l'information complexe et la culture des connaissances.

\begin{abstract}
Formerly, creativity was not a pedagogical priority. Today however, teachers and artists reinvent themselves as « geeks » and « designers ». Increasingly, scientific models are giving a new shape to « creativity » on the Web, fostering a combination of « mediation » and « mediatisation» and promoting a repertoire of e-strategies for creative literacies. This renewal of the creative studies has an impact on the Humanities for they are invited to a renewed interdisciplinary cooperation. In this context, Transliteracy defined as the convergence between media literacy, information literacy and computer literacy, can lead to empower-
\end{abstract}


ment where the benefits of new cognitive learning modes are shared, learner-centered and not just technology-driven with the prospect of a SMILE alliance, that is Synergies for Media and Information Literacies built on the multi-stakeholder governance required by complex information and knowledge cultures. 
